\title{
Method for detecting vertical offset of coupling coil using signal coil based on magnetic coupling resonance wireless power transfer system
}

\author{
Ye Zhaohong ${ }^{1}$, Gao Yuan ${ }^{1, *}$ \\ ${ }^{1}$ College of Automation, Chongqing University, Chongqing 400044, China
}

\begin{abstract}
To improve the reliability in wireless power transfer system, communication is widely used. An independent coil as the signal coil for data transfer has the advantage of decoupling power coupling mechanism and signal coil when designing the system. Besides, in some application, vertical offset of the power coil may impact the stability of the system, position detection of the receiver side power coil is needed. Method of using the signal coil for vertical offset detection is adopted in this paper, simulation result verified the feasibility. The vertical offset of the power coil can be estimated by an exponential function based on the system parameter and the current of the primary side power coil. The exponential fitting function used in the paper can be used as a reference when designing the detection.
\end{abstract}

\section{Introduction}

Wireless power transfer (WPT) technology has been widely applied for the advantage of convenience and safety compared with cable transfer [1-3]. Recently, in the field of consumer electronics, the demand of wireless charging increased dramatically [4-6]. To realize high performance power transmission, the communication is indispensable. As a result, simultaneous wireless power and information transfer (SWPIT) is vital to reach reliable data transfer as it can help to monitor and control the outputs [7-10]. A separate signal transmission technology has the merits of decoupling the power transfer and communication, but an additional signal coil is required [11]. Meanwhile, it is vital to get the position of the secondary power coil to guarantee a reliable energy transfer. The traditional method is using infrared sensors, or the current signal of the primary side to get offset information. In this paper, a method for detecting the deviation of the secondary power coil in the vertical direction is proposed by using the communication coil. A circular coupling mechanism and a suitable signal coil is designed for verification. Finite element method (FEM) is used to analyse and optimize the size of the signal coil to ensure stable signal transmission while maximizing the performance of the vertical offset detection.

\section{System Overview}

Fig. 1 depicts the system schematic diagram. The input matching circuit convert the $50 \mathrm{~Hz} \mathrm{AC}$ sine voltage from the grid into $110 \mathrm{~V}$ DC bus voltage. And the DC power is inverted into $150 \mathrm{kHz}$ high frequency $\mathrm{AC}$ square voltage by the inverter. The compensation network follows the inverter drive the primary power coil and transform the electrical energy into magnetic energy. The secondary side power coil induces a voltage in the high-frequency magnetic field, and the compensation network together with the rectifier circuit outputs a stable DC power to supply the load (circuit). The signal coil is placed between the power coils, which is connected to the signal processing circuit for realizing signal transmission and detecting the vertical offset of the power coil.

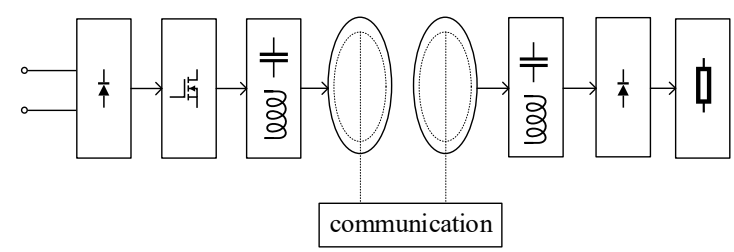

Fig. 1. The schematic diagram of the system

\section{1 power transfer design}

The core part of the magnetic coupling resonance wireless power transfer (MCR-WPT) system is the coupling mechanism, which determines the energy transfer performance of the system. Commonly used plane type coupling mechanisms are round, square, rectangular, etc. This paper, a planar circular coupling mechanism is designed, and the power transfer distance is $20 \mathrm{~mm}$. The primary side and secondary side of the coupling mechanism is designed to be symmetrical.

\footnotetext{
* Corresponding author: gaoyaun99@cqu.edu.cn
} 


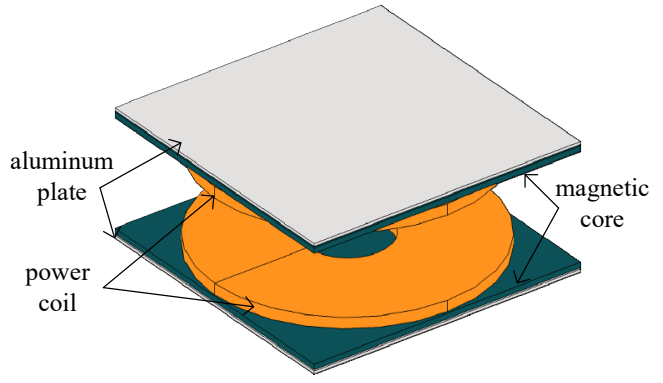

Fig. 2. 3D simulation model

In Fig. 2, the primary side and secondary side of the coupling mechanism each contain three parts, which are the energy coil (outer diameter $100 \mathrm{~mm}$, inner diameter $33 \mathrm{~mm}$, 2 layers, 14 turns/layer, thickness $5 \mathrm{~mm}$ ), the magnetic core (length $100 \mathrm{~mm}$, width $100 \mathrm{~mm}$, height $2 \mathrm{~mm}$ ) and the aluminium shielding board (length $100 \mathrm{~mm}$, width $100 \mathrm{~mm}$, height $2 \mathrm{~mm}$ ). The magnetic core is used for increasing coupling coefficient to improve the energy transmission efficiency. The aluminium plate is used for shielding the magnetic leakage of the coupling mechanism which can help to reduce the impact of the coupling mechanism on the subsequent circuit of the system. Obviously, regardless of the angle of plane rotation, the coupling performance of the coupling mechanism hardly changes. The parameters of the designed coupling mechanism are shown in Table 1.

Table 1. Specification of the coupling mechanism

\begin{tabular}{cc}
\hline Parameters & Designed value \\
\hline coil inductance $\left(L_{p}\right)$ & $80.92 \mu \mathrm{H}$ \\
coil DC resistance $\left(R_{p}\right)$ & $32.87 \mathrm{~m} \Omega$ \\
coil inductance $\left(L_{s}\right)$ & $80.29 \mu \mathrm{H}$ \\
coil DC resistance $\left(R_{s}\right)$ & $32.42 \mathrm{~m} \Omega$ \\
Mutual inductance $(M)$ & $36.33 \mu \mathrm{H}$ \\
Coupling coefficient $(k)$ & 0.449 \\
\hline
\end{tabular}

To clarify the vertical offset characteristics of the coupling mechanism. Based on Fig. 2, by using FEM simulation, the relationship between the mutual inductance of the two power transfer coils and the vertical offset distance is shown in Fig. 3. Negative value means closer of the distance, positive means farther. It can be obtained that the mutual inductance of the coils and vertical offset is approximately exponential relationship.

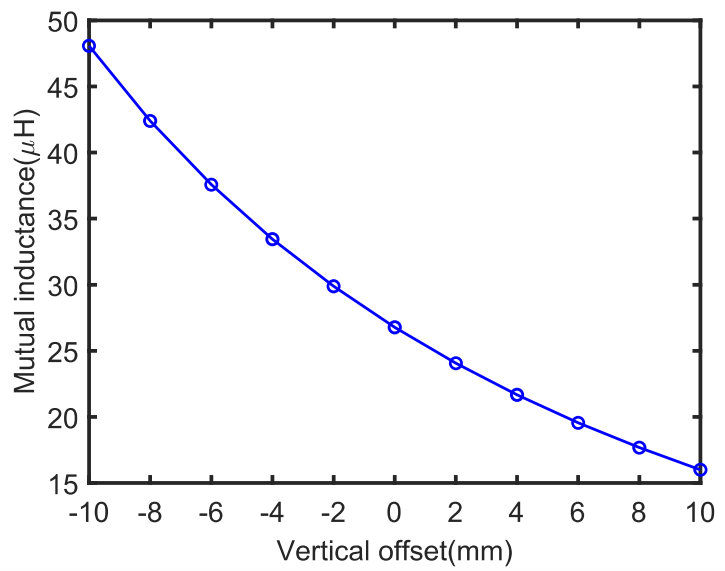

Fig. 3. Relationship between mutual inductance and offset
Considering the power variance and open loop of the receiving side at any time. To ensure the safe and stable operation of the system, an LCC-S compensation topology is selected as shown in Fig. 4. With this type compensation, the current of the primary side power coil is constant and the output voltage of the secondary side power coil is constant no matter of the load variance. Besides, the main part of the MCR-WPT system is on the primary side, which simplifies the structure of the secondary side circuit, and makes it lightweight.

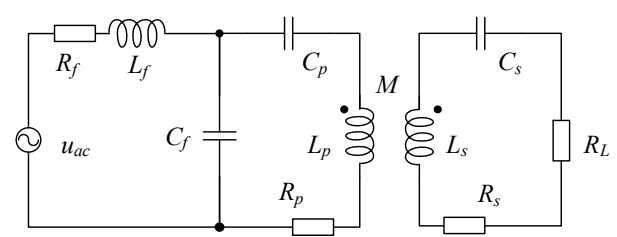

Fig. 4. LCC-S compensation topology

In Fig.4, the parameter's value should meet:

$$
\begin{gathered}
\omega_{0}^{2} L_{f} C_{f}=1 \\
\omega_{0}^{2}\left(L_{p}-L_{f}\right) C_{p}=1 \\
\omega_{0}^{2} L_{s} C_{s}=1
\end{gathered}
$$

Where, $\omega_{0}$ is the working angular frequency of the coupling system, $L_{p}$ and $L_{s}$ are the self-inductance of the primary and secondary power coils, $R_{p}$ and $R_{s}$ is the internal resistance, respectively. $L_{f}$ is the compensation inductance of the primary coil, $R_{f}$ is its internal resistance, and $C_{p}, C_{f}$, and $C_{s}$ are the compensation capacitors. The parameters' value used in this paper is shown in Table 2.

Table 2. Specification of the compensation component

\begin{tabular}{cc}
\hline Parameters & Value \\
\hline$C_{f}$ & $29.02 \mathrm{nF}$ \\
$C_{p}$ & $26.73 \mathrm{nF}$ \\
$C_{s}$ & $14.02 \mathrm{nF}$ \\
$L_{f}$ & $38.80 \mu \mathrm{H}$ \\
\hline
\end{tabular}

By adjusting the primary side power to compensate for energy fluctuation caused by vertical offset, and the output power performance of the system can be improved. To excite the compensation topology shown in Fig.4, a voltage-type full-bridge inverter circuit shown in Fig.5 is used, the DC bus voltage $U_{d c}$ is $110 \mathrm{~V}$, the inverter frequency is $150 \mathrm{kHz}$, and the switching tube duty cycle is around $50 \%$.

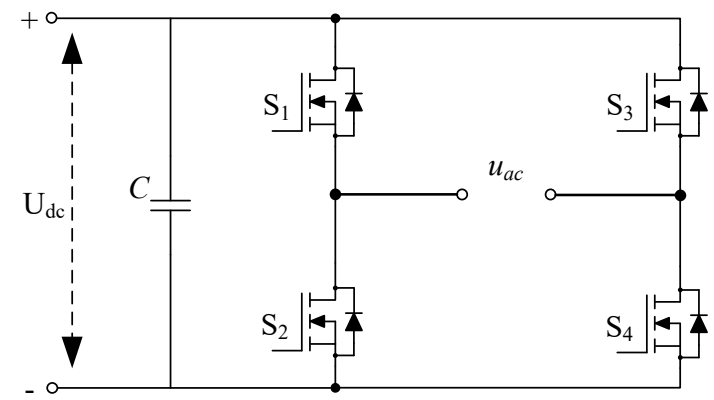

Fig. 5. The circuit topology of power driver 


\section{2 signal transfer design}

The coupling mechanism converts the high frequency AC current into magnetic energy. In Fig. 6, the magnetic field distribution based on FEM shows that the magnetic field generated by the circular mechanism in the threedimensional space has a regular distribution.

The magnetic field distribution in the plane parallel to the power coil is a shape of gradual ring. And Fig. 7 manifests that the magnetic field is symmetrically distributed on the plane horizontally.

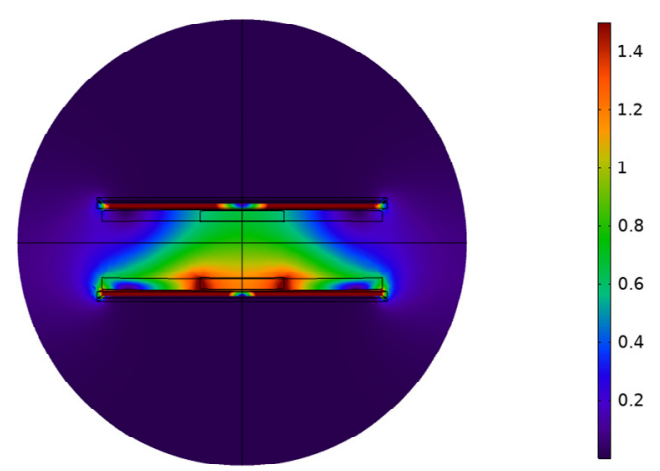

Fig. 6. Magnetic field of the coupling mechanism (unit mT)

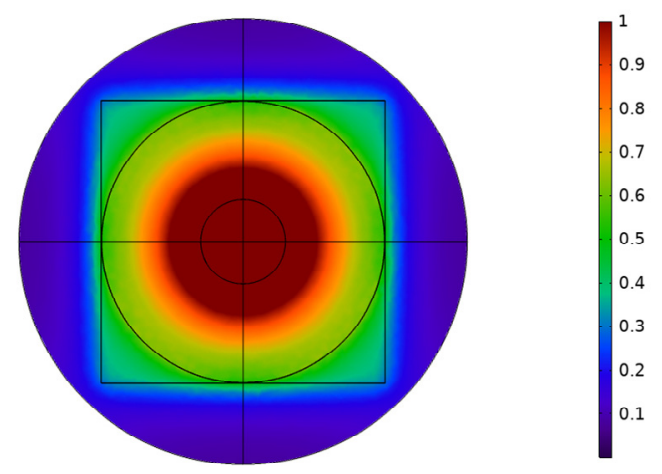

Fig. 7. Magnetic field distribution horizontal (unit $\mathrm{mT}$ ) $2 \mathrm{~mm}$ offset from the surface of the power coil

The performance of the signal transmission is affected by the induced voltage generated by the power magnetic field. In addition, the high frequency harmonic noise generated by the switching tube of the inverter will also cause large electromagnetic interference to the signal transmission. Based on the analysis above, signal coils with DD structure is commonly used to reduce the interference of power magnetic field. The DD coil mean two D shape coil in series. The winding method of the DD coil structure is shown in Fig. 8. The signal coil made of enamelled copper wire contain only one turn and the two semi-circular D-shaped coils are designed in axial symmetry, and the circuit relationship is in series. According to Fig. 7 and Fig. 8, since the magnetic field in the plane where the signal coil is located is symmetrically distributed, and the symmetry axis of the magnetic field coincides with the symmetry axis of the signal coil. The reference directions of the two D-shaped coils are different, thus the induced voltages of the two signal coils are the same in magnitude and opposite in direction. The port voltage of the DD signal coil is zero. The interference of the energy magnetic field on the two D shape signal coil will cancel each other out. For the convenience of description, a quantity $\alpha$ can be defined, which normalizes the size of the signal coil, and represents the ratio between the diameter of the signal coil and the outer diameter of the energy coil.

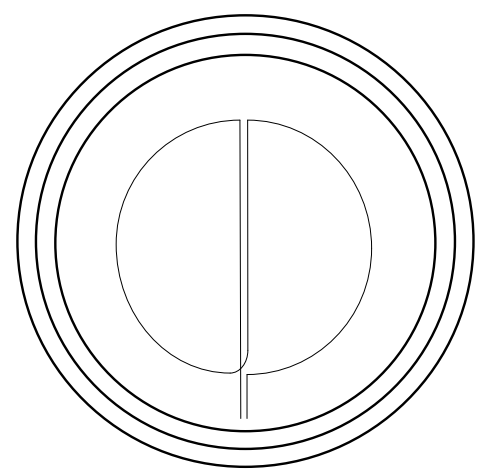

Fig. 8. Top view of the power and signal coil

An FEM based simulation model is built shown in Fig. 10. The relationship between the induced voltage of the Dshape signal coil and the current of the power coil can be obtained by exciting the primary side power coil with constant current. Thus, the vertical offset of the coupling mechanism can be obtained according to the magnitude of the induced voltage on the D-shaped signal coil.

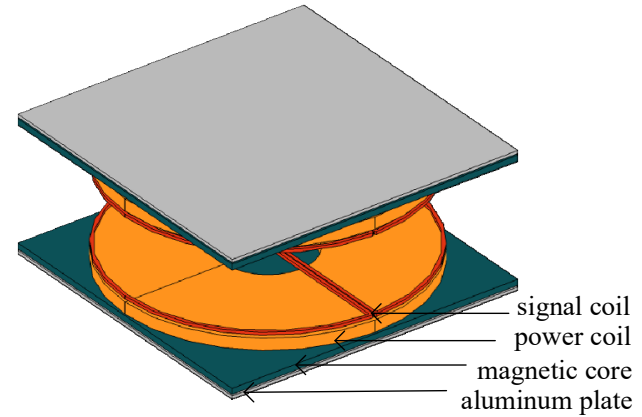

Fig. 9. Placement of the coupling mechanism with signal coil

\section{3 signal coil optimization}

Metal in the magnetic field will produce eddy current effects, which will affect the performance of the coupling. The signal coils made of metal may also decrease the performance of the coupling mechanism. To minimize the influence of signal coil on power transfer, it is necessary to reduce the volume and size of the signal coil while ensuring reliable signal transmission. In addition, in highfrequency circuits, the radial depth of the current that the alternating current can reach along the surface of the wire is limited. Thence, it is necessary to study the actual effective area for improving the total performance. The calculation formula of depth is:

$$
\Delta=\sqrt{\frac{2}{\omega \mu \gamma}}
$$

Where, $\Delta$ is the penetration depth $(\mathrm{m}), \omega$ is angular frequency, $\mu$ is permeability, and $\gamma$ is conductivity. While the signal coil is made of copper, the relationship between 
the current skin depth of the wire and the current frequency is shown in Fig. 11.

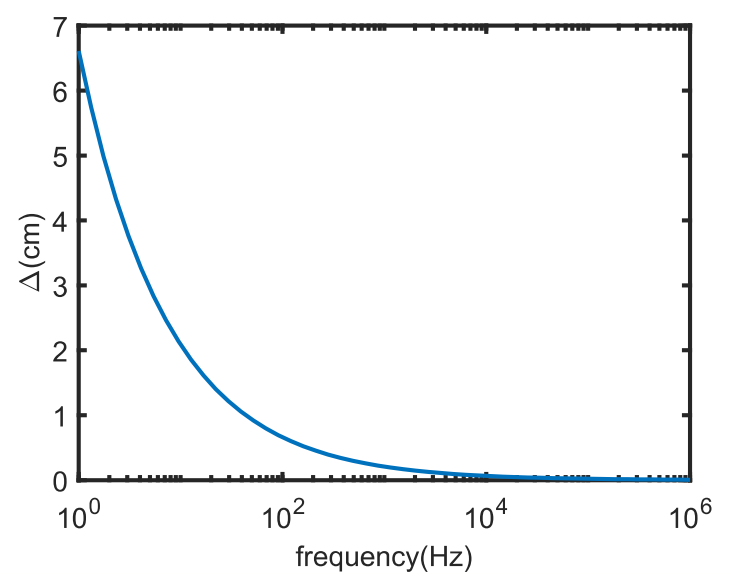

Fig. 10. Relationship of the penetration depth and frequency

According to Fig. 10, as frequency become higher, the penetration depth became shallower. The frequency of current in the power coil is much lower than signal frequency, so only the effect of the signal coil current should be considered when designing the system. Considering current intensity, the current capability of the signal coil can also be increased by means of two-wire or multi-wire parallel winding. As shown in the Fig. 10, the designed PCB signal coil has a diameter of $97 \mathrm{~mm}$, a trace width of $10 \mathrm{mil}$, and a copper foil process of $1 \mathrm{OZ}$. To ensure the continuity of the current, the arc trace is used at the corner. To ensure the symmetry of the left and right coils, the signal coil traces are on the same layer without cross-layers. The coil ports are connected to the signal modem using twisted wire pair.

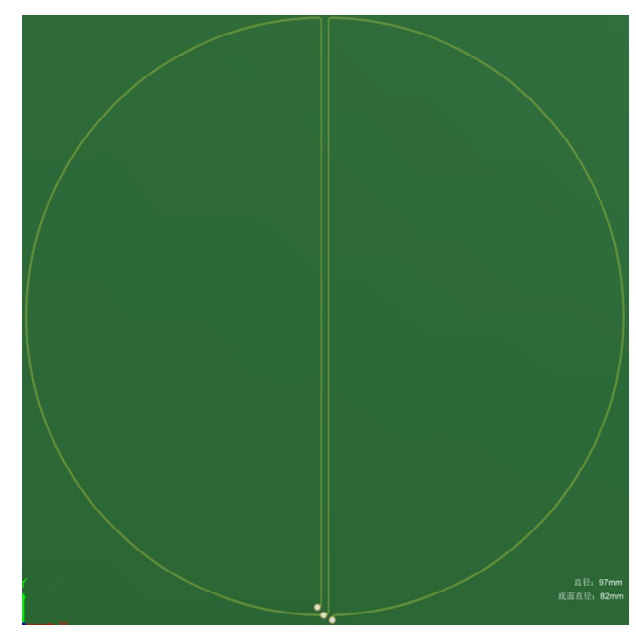

Fig. 11. The PCB photo of a DD signal coil

\section{Results and Conclusions}

Power transfer distance between the primary and secondary side coils is $20 \mathrm{~mm}$, with no offset horizontally and vertically. The excitation current on the primary power coil is 1A, and the secondary side is open loop. The relationship between the magnitude of the induced voltage of the single-turn D shape coil and the size of the coil is shown in Fig. 12. It is indicated that as the size of the coil increases, the induced voltage increases and then decreases, and the only extreme point is obtained near the radius of 0.85 . For the DD coil, there is no obvious relationship between the voltage and its size, but statistically it has a slight upward trend. Obviously, the voltage of the DD coil is more than 1000 times smaller than the voltage value of the $\mathrm{D}$ coil. For the results below, an $\alpha=0.82$ coil is used.

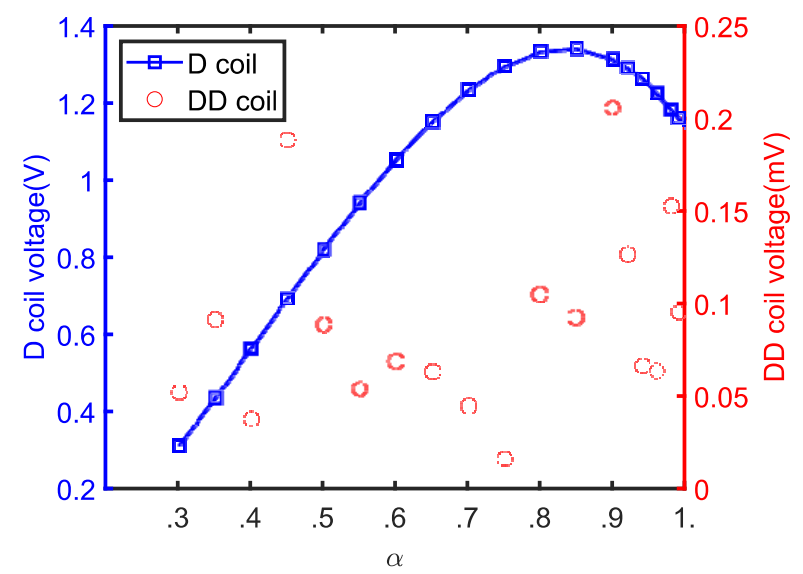

Fig. 12. Relationship between the coil voltage and its size

To figure out the regular pattern between the induced electromotive force of the single-turn signal coil and its size. The excitation current on the primary side power coil is $1 \mathrm{~A}$, and the secondary side power coil is open loop. The relationship between the induced voltage of the primary signal coil and the vertical transmission distance offset is shown in Fig. 13. It can be seen that when the excitation current of the primary coil is fixed, the induced voltage of the primary $\mathrm{D}$ shape signal coil has an exponential relationship with the vertical offset distance.

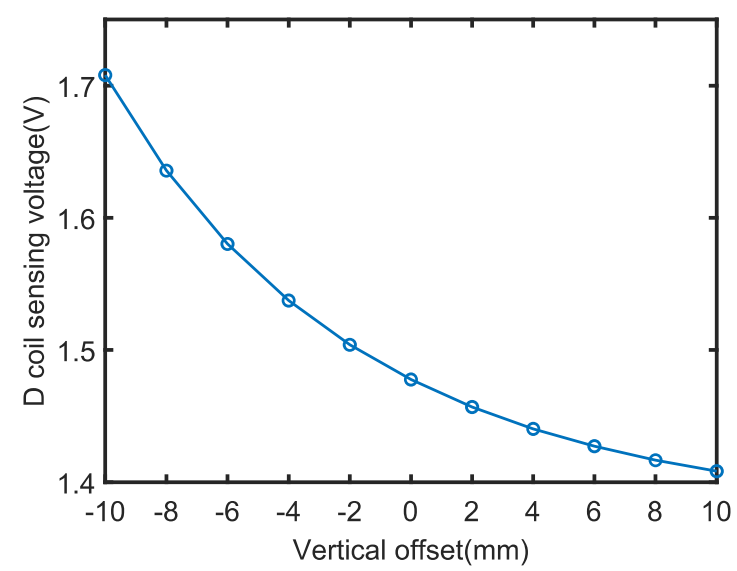

Fig. 13. Induced voltage and vertical offset

Fit the data plot using an exponential model:

$$
f(\mathrm{x})=\mathrm{a} \cdot \exp (-\mathrm{b} \cdot \mathrm{x})+\mathrm{c}
$$

The fitting result is $\mathrm{a}=0.0964, \mathrm{~b}=-0.122, \mathrm{c}=1.381$. The fitting performance is shown in Table 3. According to the table, the fitting is very accurate.

Table 3. Indicators of the fitting

\begin{tabular}{cc}
\hline Criterion & Value \\
\hline SSE & $3.97 \mathrm{E}-6$ \\
R-square & 1 \\
\hline
\end{tabular}




\begin{tabular}{cc}
\hline Adjusted R-square & 0.9999 \\
RMSE & $7.05 \mathrm{E}-4$ \\
\hline
\end{tabular}

To figure out the influence of the horizontal offset on the signal coil, the excitation current of the primary power coil is set to be fixed at $1 \mathrm{~A}$, and the secondary power coil is open loop. The result is obtained in Fig. 14, compared with Fig. 13, the influence of the horizontal offset on the signal coil is much smaller than the vertical offset.

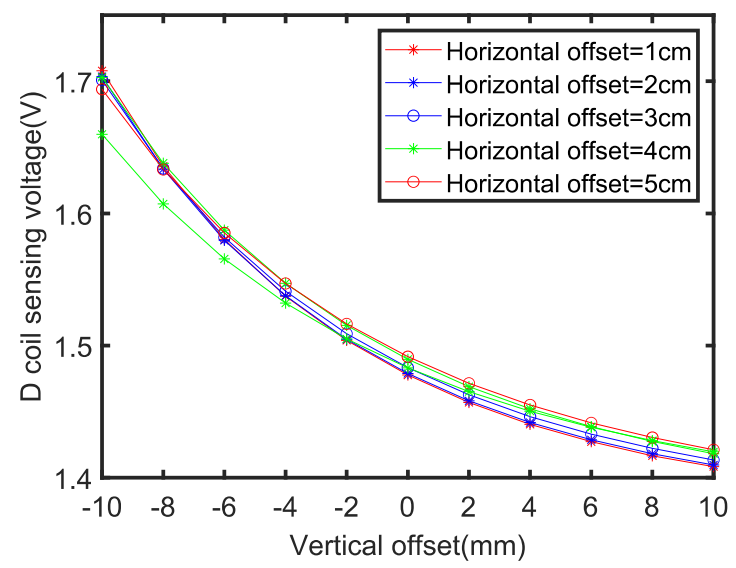

Fig. 14. The influence of horizontal offset

By exciting the primary energy coil with currents of different magnitudes and keeping the secondary energy coil open, multiple sets of results as shown in Fig. 15 can be obtained. And the exponential function model is used to fit the data set, the fitting results are shown in Table 5.

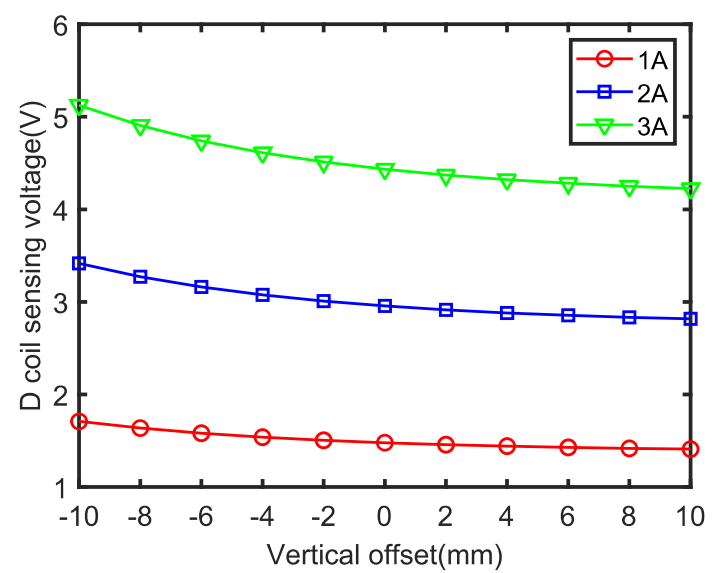

Fig. 15. Power coil excited by different current

Table 4. Parameter values and fitting performance

\begin{tabular}{cccc}
\hline Parameter & $1 \mathrm{~A}$ & $2 \mathrm{~A}$ & $3 \mathrm{~A}$ \\
\hline $\mathrm{a}$ & 0.0964 & 0.1928 & 0.2891 \\
$\mathrm{~b}$ & 0.122 & 0.122 & 0.122 \\
$\mathrm{c}$ & 1.381 & 2.761 & 4.142 \\
SSE & $3.97 \mathrm{E}-6$ & $1.56 \mathrm{E}-5$ & $3.59 \mathrm{E}-6$ \\
\hline
\end{tabular}

The voltage induced on the $\mathrm{D}$ coil is positively correlated with the excitation current on the primary coil, under other conditions fixed, according to Fig. 15 and Table 4, it can be wrote as below:

$$
f(\mathrm{x})=[\mathrm{a} \cdot \exp (-\mathrm{b} \cdot \mathrm{x})+\mathrm{c}] \cdot \mathrm{I}_{\mathrm{p}}
$$

Where, $a, b$ and $c$ are the fitting result of the plot in Fig. 15 and $I_{p}$ is the current of the primary side power coil. To realize the parameter above, once the system is setup, one can get a set of data in Fig. 13, and then fit the plot using equation (6), and program the data into the MCU control chip. Based on the analysis and results above, it can be seen that fixing the secondary side power coil open loop, when the primary side coil current is known, the vertical offset of the secondary side power coil can be obtained by the magnitude of the induced voltage on the primary side signal coil.

\section{Reference}

1. Wu, J., et al., A Coupling Mechanism With Multidegree Freedom for Bidirectional Multistage WPT System. IEEE Trans. Power Electron., 36(2): p. 1376-1387 (2021)

2. Ustun, D., S. Balci, and K. Sabanci, A parametric simulation of the wireless power transfer with inductive coupling for electric vehicles, and modelling with artificial bee colony algorithm. Measurement, 150 (2020)

3. Rasekh, N., et al., Thermal analysis and electromagnetic characteristics of three single-sided flux pads for wireless power transfer. J. Clean. Prod.,243 (2020)

4. Yang, L., et al., Analysis and Design of Three-Coil Structure WPT System With Constant Output Current and Voltage for Battery Charging Application. Ieee Access, 7: p. 87334-44 (2019)

5. Yang, C., et al., Analysis, design and implement of asymmetric coupled wireless power transfer systems for unmanned aerial vehicles. AIP ADV, 9(2) (2019)

6. Liu, S., X.M. Li, and L. Yang, Three-coil structurebased WPT system design for electric bike CC and CV charging without communication. IET ELECTR POWER APP, 13(9): p. 1318-1327 (2019)

7. Sun, Y.S., et al., Bidirectional Simultaneous Wireless Information and Power Transfer via Sharing Inductive Link and Single Switch in the Secondary Side. Ieee Access, 8: p. 184187-98 (2020)

8. Li, Y.L., X.F. Li, and X. Dai, A Simultaneous Wireless Power and Data Transmission Method for Multi-Output WPT Systems: Analysis, Design, and Experimental Verification. Ieee Access, 8: p. 206353206359 (2020)

9. Li, X.F., et al., A Decoupled Power and Data-Parallel Transmission Method With Four-Quadrant Misalignment Tolerance for Wireless Power Transfer Systems. IEEE Trans. Power Electron., 34(12): p. 11531-11535 (2019)

10. Kassab, H., J. Louveaux, and Ieee. Simultaneous Wireless Information and Power Transfer Using Rectangular Pulse and CP-OFDM. IEEE International Conference on Communications. Shanghai, PEOPLES R CHINA (2019) 
11. Bieler, T., M Perrottet, V Nguyen, et al.

Contactless power and information transmission.

IEEE Trans. Ind. Appl., 38(5) : 1266-72 (2002) 